\title{
A 3-D MOMENT BASED APPROACH FOR BLOOD VESSEL DETECTION AND QUANTIFICATION IN MRA
}

P. Reuzé( $\left.{ }^{*}\right)$, J.L. Coatrieux ${ }^{(*)}$, L.M. Luo ${ }^{(*)}$, J.L. Dillenseger ${ }^{(*)}$

(*) Laboratoire Traitement du Signal et de l'Image

Campus de Beaulieu, Université de Rennes I, 35042 Rennes Cédex, France.

Tél : 33-99-28-62-20

Fax : 33-99-28-69-17

(**) Vision and Image Processing Laboratory

Southeast University, Nanjing, China. 


\begin{abstract}
This paper describes a new method for the three-dimensional (3-D) tracking and the quantification of blood vessels from Magnetic Resonance Angiography (MRA). The approach is based on the 3D geometrical moments and consists of the following steps: (1) interactive selection of 3-D seed points; (2) automatic tracking of the vessels; (3) local computation of both diameter and orientation; (4) rendering of the vessels. This detection and estimation scheme has been validated on simulated and real data.
\end{abstract}

Key Words : Magnetic Resonance Angiography, detection, quantification, tracking, rendering. 


\section{Introduction}

It is well known that the capability to image the vascular network is of critical importance for the diagnosis of vascular abnormalities, pre-surgical evaluation and planning as well as tumor assessment and analysis. Several imaging modalities are capable to bring the suitable clinical information. They differ in the physical principles (X-ray, MRI), the dimensional space they provide (2-D versus $3-\mathrm{D}$ ), the spatial and temporal resolutions (static and dynamic), the invasive or non-invasive nature and the use or not of a contrast medium.

The most common source is still the X-ray angiography which is widely applied for moving and non-moving structures. Despite their high spatial and temporal resolutions, the need to make use of a dye-product and the basic projective-integrative rule for the image formation are two main limitations. However, some attempts have been recently made to recover the 3-D information from two or three image projections [1] [2] or from temporal image sequences through motion estimation when a moving organ is observed [3]. The feasibility of a 3-D X-Ray scanner has been demonstrated since the pioneering device, the Dynamic Spatial Reconstructor (DSR) [4]. Automatic segmentation of the coronary arterial tree from 3-D DSR angiogram is proposed in [5], where the detection of seed points being performed, a region growing after sigma filtering is applied. A new design and prototyping of 3-D CT scanner has been recently undertaken which allows the reconstruction of high contrast tissues such as bones and vessels [6].

The development of Magnetic Resonance Angiography (MRA) provides another competitive way to define in 3-D the vessel networks without requiring contrast medium. This advanced technique for non-invasive vessel imaging, as the previous ones, is of interest as soon as a precise detection and a full 
characterization can be carried out. The two information that must be looked for are the 3-D local orientation of the vessel and the diameter. A first approach for enhancement and segmentation has been proposed in [7], based on a non-linear diffusion model followed by a convolution with derivations of gaussians using multiple orientations. Markov Random Field models have also been applied [8], but they are computationally intensive. Recently Trousset [9] proposed an algorithm for vessel tracking in 3D angiograms, which needs starting and ending points and a given starting direction. This algorithm is an extension of a 2-D tracking described in [10].

A moment-based algorithm is here described. The vessel is locally approximated by a cylinder whose orientation and diameter are estimated within a spherical window of variable size ( 3,5 and 7 voxels). The interactive selection of seed points in 2-D (orthogonal slice viewing) or 3-D (Maximum Intensity Projection, MIP) leads to avoid a full image traversal and to reduce significantly the computation time.

The next paragraph describes the vessel model and the estimation method. The decision step is then reported. A quantitative validation (section III) is carried out on synthetic cylinders with additive noise and on real data coming from low field MRA. The visual evaluation of the results is performed by means of the laboratory designed 'X-Image 3-D' software [11].

\section{Characterization of a vessel}

\section{II.1) Definition of moments}

Moments and functions of moments have been widely used for image analysis and object representation (refer to [12] for a survey). In an early paper, $\mathrm{Hu}$ [13] has shown that if a function is piece wise continuous and has non zero values only in a finite region then moments of all order exist. 
The three dimensional moments of order $\mathrm{p}+\mathrm{q}+\mathrm{r}$ of a density distribution function $\mathrm{f}(\mathrm{x}, \mathrm{y}, \mathrm{z})$ is defined as :

$$
M_{p q r}=\iiint_{V} x^{p} y^{q} z^{r} f(x, y, z) d x d y d z
$$

The first order moments are used to localize the center of mass of the object in a given window. Its coordinates are expressed by :

$$
\overline{\mathrm{x}}=\frac{\mathrm{M}_{100}}{\mathrm{M}_{000}} \quad \overline{\mathrm{y}}=\frac{\mathrm{M}_{010}}{\mathrm{M}_{000}} \quad \overline{\mathrm{z}}=\frac{\mathrm{M}_{001}}{\mathrm{M}_{000}}
$$

In the tracking algorithm, the window is centered on the voxel closest to the center of mass. In other words, the sum $\left(\overline{\mathrm{x}}^{2}+\overline{\mathrm{y}}^{2}+\overline{\mathrm{z}}^{2}\right)$ is minimized in order to have the best fit between the vessel and the model. The modified moments are then computed :

$$
\mu_{\mathrm{pqr}}=\iiint_{\mathrm{V}}(\mathrm{x}-\overline{\mathrm{x}})^{\mathrm{p}}(\mathrm{y}-\overline{\mathrm{y}})^{\mathrm{q}}(\mathrm{z}-\overline{\mathrm{z}})^{\mathrm{r}} \mathrm{f}(\mathrm{x}, \mathrm{y}, \mathrm{z}) \mathrm{dxdydz}
$$

The relations between centered moments, $\mu_{\mathrm{pqr}}$, and original moments, $\mathrm{M}_{\mathrm{pqr}}$, for second order are given by :

$$
\begin{aligned}
& \mu_{110}=M_{110}-\frac{M_{100} M_{010}}{M_{000}} \\
& \mu_{011}=M_{011}-\frac{M_{010} M_{001}}{M_{000}} \\
& \mu_{101}=M_{101}-\frac{M_{100} M_{001}}{M_{000}} \\
& \mu_{200}=M_{200}-\frac{\left(M_{100}\right)^{2}}{M_{000}} \\
& \mu_{020}=M_{020}-\frac{\left(M_{010}\right)^{2}}{M_{000}} \\
& \mu_{002}=M_{002}-\frac{\left(M_{001}\right)^{2}}{M_{000}}
\end{aligned}
$$




\section{II.2) Modeling}

A vessel is locally modeled by a cylinder of orientation $\alpha$ and $\beta$ and diameter $\mathrm{d}$ (Figure 1a). A Cartesian coordinate system (E1) is used, where $\alpha$ is the angle between the projection on Oxy of the axis cylinder and $O x$, and $\beta$ is the angle between the axis of the cylinder and Oz. The vessel intensity is denoted Ic and the background intensity, $\mathrm{I}_{\mathrm{b}}$, with $\mathrm{I}_{\mathrm{c}}>\mathrm{I}_{\mathrm{b}}$.

\section{II.3) Computation of the orientation}

A rotation is performed to align the cylinder axis with $\mathrm{Oz}$ (Figure $1 \mathrm{~b}$ ) and the moments become:

$$
\mu_{\mathrm{pqr}}^{\prime}=\iint_{\mathrm{v}} \begin{gathered}
(\mathrm{x} \cdot \cos \alpha \cdot \cos \beta+\mathrm{y} \cdot \sin \alpha \cdot \cos \beta-\mathrm{z} \cdot \sin \beta)^{\mathrm{p}} \cdot(-\mathrm{x} \cdot \sin \alpha+\mathrm{y} \cdot \cos \alpha)^{\mathrm{q}} \\
\cdot(\mathrm{x} \cdot \cos \alpha \cdot \sin \beta+\mathrm{y} \cdot \sin \alpha \cdot \cos \beta+\mathrm{z} \cdot \cos \beta)^{\mathrm{r}} \cdot \mathrm{f}(\mathrm{x}, \mathrm{y}, \mathrm{z}) \cdot \mathrm{dxdydz}
\end{gathered}
$$

From (5), the following equations can be derived [14 ] :

$$
\begin{aligned}
\mu_{020}^{\prime}= & \mu_{200} \sin ^{2} \alpha+\mu_{020} \cos ^{2} \alpha-\mu_{110} \sin 2 \alpha \\
\mu_{002}^{\prime}= & \left(\mu_{200} \cos ^{2} \alpha+\mu_{020} \sin ^{2} \alpha+\mu_{110} \sin 2 \alpha\right) \sin ^{2} \beta+\mu_{002} \cos ^{2} \beta+ \\
& \left(\mu_{101} \cos \alpha+\mu_{011} \sin \alpha\right) \cdot \sin 2 \beta
\end{aligned}
$$

Where $\mu_{002}^{\prime}$ is maximum with respect to $\alpha$ and $\beta, \mu_{020}^{\prime}$ and $\mu_{002}^{\prime}$ being minimum. Searching extrema in $\mu_{020}^{\prime}$ and $\mu_{002}^{\prime}$ leads to the two angles :

$$
\alpha_{0}=\frac{1}{2} \arctan \left(\frac{2 \mu_{110}}{\mu_{200}-\mu_{020}}\right)+\mathrm{k} \frac{\pi}{2}
$$




$$
\beta_{0}=\frac{1}{2} \arctan \left(\frac{2 \mu_{101} \cos \alpha_{0}+\mu_{011} \sin \alpha_{0}}{\mu_{002}-\mu_{200} \cos ^{2} \alpha_{0}-\mu_{200} \sin ^{2} \alpha_{0}-\mu_{110} \sin 2 \alpha_{0}}\right)_{+\mathrm{k}} \frac{\pi}{2}
$$

The final values of $\alpha_{0}$ and $\beta_{0}$ (e.g. parameter $\mathrm{k}$ ) are obtained through the minimization of $\mu_{020}^{\prime}$ and the maximization of $\mu_{002}^{\prime}$.

\section{II.4) Computation of the diameter}

The computation of moments is performed in a spherical window. Under the assumption of having two homogeneous regions it can be shown that:

$$
\mathrm{M}_{000}=\mu_{000}=\iiint_{\mathrm{V}_{\mathrm{S} \cap \mathrm{C}}} \mathrm{I}_{\mathrm{C}} \mathrm{dv}+\iiint \int_{\mathrm{V}_{\mathrm{S}}-\mathrm{V}_{\mathrm{S} \cap \mathrm{C}}} \mathrm{I}_{\mathrm{b}} \mathrm{dv}
$$

where $V_{\mathrm{s} \cap \mathrm{C}}$ is the volume of the cylinder in the sphere, and Vs is the volume of the sphere. The diameter $d$ is then [15] :

$$
\mathrm{d}=\mathrm{n}\left[1-\left(\frac{\mu_{000} \frac{3}{4 \pi}-\mathrm{I}_{\mathrm{c}}}{\mathrm{I}_{\mathrm{b}}-\mathrm{I}_{\mathrm{c}}}\right)^{2 / 3}\right]^{1 / 2}
$$

with $\mathrm{n}$, the size of the window in voxels.

\section{II.5) Estimation of intensities}

The intensities of the vessel $\left(\mathrm{I}_{\mathrm{c}}\right)$ and the background $\left(\mathrm{I}_{\mathrm{b}}\right)$ are unknown. These values cannot be considered constant in the whole image (because of MRI artifacts and non stationarities).

At a given seed point, the value of the mean intensity along the estimated vessel direction, and the estimated mean gray level computed around the vessel allow to initialize $I_{c}$ and $I_{b}$ respectively. 
Assuming smooth variations of intensities during the tracking, these values can be updated at site s by making use of the estimations at site s-1 as follows :

$$
\begin{aligned}
& \hat{\mathrm{I}}_{\mathrm{c}}^{\mathrm{s}}=\mathrm{a}_{0} \mathrm{I}_{\mathrm{c}}^{\mathrm{s}}+\left(1-\mathrm{a}_{0}\right) \hat{\mathrm{I}}_{\mathrm{c}}^{\mathrm{s}-1} \\
& \hat{\mathrm{I}}_{\mathrm{b}}^{\mathrm{s}}=\mathrm{a}_{0}^{\prime} \mathrm{I}_{\mathrm{b}}^{\mathrm{s}}+\left(1-\mathrm{a}_{0}^{\prime}\right) \hat{\mathrm{I}}_{\mathrm{b}}^{\mathrm{s}-1}
\end{aligned}
$$

where $\mathrm{a}_{0}$ and $\mathrm{a}_{0}$ are weighting coefficients. When fast variations of the intensities are present, high values of $\mathrm{a}_{0}$ and $\mathrm{a}_{0}^{\prime}$ must be chosen.

\section{II.6) Discrete computation}

The above equations used above are applied to a continuous 3-D function $\mathrm{f}(\mathrm{x}, \mathrm{y}, \mathrm{z})$. However MRA images provide a set of elementary volumes (voxels) arranged on an isotropic discrete grid.

So, equation (1) becomes :

$$
\begin{gathered}
\mathrm{M}_{\mathrm{pqr}}=\sum \sum \sum{ }_{\mathrm{i}}{ }^{\mathrm{j}} . \mathrm{j}^{\mathrm{q}} . \mathrm{k}^{\mathrm{r}} . \mathrm{f}_{\mathrm{d}}(\mathrm{i}, \mathrm{j}, \mathrm{k}) \\
\text { with } \mathrm{f}_{\mathrm{d}}(\mathrm{i}, \mathrm{j}, \mathrm{k})=\int_{\mathrm{i}-1 / 2}^{\mathrm{i}+1 / 2} \int_{\mathrm{i}-1 / 2}^{\mathrm{i}+1 / 2} \int_{\mathrm{i}-1 / 2}^{\mathrm{i}+1 / 2} \mathrm{f}(\mathrm{x}, \mathrm{y}, \mathrm{z}) \mathrm{dxdydz}
\end{gathered}
$$

To approximate the spherical window, a decomposition is performed by dividing each voxel in $\mathrm{mx} \mathrm{mx}$ sub-voxels. A function $\mathrm{X}(\mathrm{x}, \mathrm{y}, \mathrm{z})$ is introduced which is equal to 1 inside the sphere and 0 outside. Equation (11) becomes :

$$
\begin{aligned}
& M_{p q r}=\sum_{i=-n / 2}^{n / 2} \sum_{j=-n / 2}^{n / 2} \sum_{k=-n / 2}^{n / 2} C(i, j, k) f_{d}(i, j, k) \\
& \text { with } C(i, j, k)=\sum_{i^{\prime}=i-m / 2}^{i+m / 2} \sum_{j=j-m / 2}^{j+m / 2} \sum_{k^{\prime}=k-m^{\prime} / 2}^{k+m / 2}\left(i^{\prime}\right)^{p}\left(i^{\prime}\right)^{q}\left(k^{\prime}\right)^{r} X\left(i^{\prime}, j^{\prime}, k^{\prime}\right)
\end{aligned}
$$

This way, the moment computation is reduced to mask convolutions. 


\section{II.7) Tracking and stopping rules}

The overall procedure is summarized Figure 2. A first point being obtained by an interactive selection on Maximum of Intensity Projection (MIP), or on 2D orthogonal slices, the direction of tracking is given by the orientation $\left(\alpha_{0}, \beta_{0}\right)$.

The decision about the presence $(\mathrm{H} 1)$ or the absence $(\mathrm{H} 0)$ of a vessel within a given window is based on a double test, or thresholding :

$$
\underset{\mathrm{H} 0}{\mathrm{I}} \stackrel{\mathrm{H} 1}{<}\left[\mathrm{I}_{\mathrm{b}}\left(1-\left(\frac{\mathrm{d}_{\min }}{2}\right)^{2}\right)^{3 / 2}+\mathrm{I}_{\mathrm{c}}\left(1-\left(1-\left(\frac{\mathrm{d}_{\min }}{2}\right)^{2}\right)^{3 / 2}\right)\right]
$$

where $d_{\min }$ is the minimal value of the diameter that has to be detected, and $\mathrm{I}=3 \mathrm{M}_{000} / 4 \pi$, the mean intensity value in the sphere. The second term represents the sum of background and vessel intensities in the window when the diameter of the vessel is $d_{\min }$. If $\mathrm{I}$ is too close to this threshold, another test is performed :

$$
\sigma_{\mathrm{H}}^{2} \underset{\mathrm{H}}{\stackrel{\mathrm{H} 1}{>}} \sigma_{\mathrm{b}}^{2}+\frac{4 \pi}{3}\left(1-\left(1-\left(\frac{\mathrm{d}_{\min }}{2}\right)^{2}\right)^{3 / 2}\right)\left(\mathrm{I}_{\mathrm{c}}^{2}-\mathrm{I}_{\mathrm{b}}^{2}\right)+\frac{4 \pi}{3} \mathrm{I}_{\mathrm{b}}^{2}-3 \frac{\mathrm{M}_{000}}{4 \pi}
$$

with $\sigma$ the standard deviation calculated in the window. The right part of (14) corresponds to the standard deviation in the window when the standard deviation of the noise is $\sigma_{b}$ and the diameter of the vessel, $d_{\min }$. If (H1) is verified, the window is centered as mentioned in (II.1) and the procedure is iterated. When the two opposite directions at a seed point have been explored, a new initial point must be entered. 


\section{Validation}

\section{III.1) Test on simulated data}

The first validation step has been carried out with computer generated cylinders of different sizes and orientations. Figure 3 depicts the sensitivity of the estimation method according to the size of the computation window. White noise, with a Signal to Noise Ratio (SNR) estimated from real data, has been added to the model.

It can be seen that the results are good as soon as the window size is greater than the object diameter. However only large windows can deal with the variations of vessel size. Unfortunately they have the inconvenient to increase the probability to find two or more vessels within the sphere. The solution consists to use an adaptive window size based on the estimations known at previous sites.

Noise robustness has also been tested (Figure 4). The error on orientation prediction is less than $5^{\circ}$ when the Signal to Noise Ratio is larger than $5 \mathrm{~dB}$. It allows the tracking to have a precision of less than one voxel.

\section{III.2) Application to real data}

The tracking algorithm has applied to MRA image sets acquired on a low field device (Magnetech, 0.1 Tesla, 64 slices, 128x128).

Figure 5 shows the result of the tracking with the algorithm described in (II.6). Twelve seed points have been selected to describe the vessels of interest. A ray tracing scheme was used to render the raw 3-D data (MIP on the left) and the resulting geometrical approximation (on the right) where the surface normal is computed from the cylindrical model. The estimation of Ic and $\mathrm{Ib}$ is done as described in (II.5). Figure 6 illustrates the better behavior that can be expected from an adaptive scheme. Intensity updating has been performed according to 
equation (10) with $\mathrm{a}_{0}=\mathrm{a}_{0}^{\prime}=0.3$ to obtain the left image while constant intensity values have been retained for the right image. It can be easily seen that the diameter of the main upper branch is overestimated when using constant values.

\section{Conclusion}

The method described above represents an efficient way to detect vascular networks in MRA. It provides the local parameters characterizing the normal or abnormal morphology of a vessel based on a cylinder-shaped model. The most interesting features appear its relative independence with the window size, its good behavior in presence of noise and the limited computation time that it requires. However, additional issues have to be considered. The first one is related to the forking situations for which the model is no more appropriate. If it seems difficult to design all the possible 3-D configurations (from bifurcation to trifurcation that are anatomically possible), at least the detection of these deviations from the model and the re initialization of the tracking procedure along these branches can be achieved. The second issue has already been mentioned : the windows must be adaptive in size to be sure that only one vessel is observed during the tracking. The last point concerns also the model: the implicit assumption is here that only two regions (object, background) are present. It may happen that three regions are involved, for example in moderate or high field MRA for cerebral arterial networks lying at the surface of the brain. A generalization of the model is then required.

Acknowledgements: The authors thank Magnetech Sopha company for providing the image sets. 


\section{REFERENCES}

[1] Garreau, M. Coatrieux, J.L. Collorec, R. Chardenon, C. (1991) A knowledgebased approach for 3-D reconstruction and labeling of vascular networks from biplane angiographic projections. IEEE trans. on Med. Imag., 10: 122131.

[2] Venaille, C. (1990) Reconstruction tridimensionnelle de réseaux vasculaires en vision trinoculaire. Thesis, Telecom Paris.

[3] Ruan, S. Bruno, A. Collorec, R. Coatrieux, J.L. (1992) 3D motion and reconstruction of coronary network. Proc. of the 14th IEEE-EMBS Conf., Paris: 2048-2049 .

[4] Ritman, E.L. Robb, R.A. Harris, L.D. (1985) Imaging Physiological Functions: Experience with the dynamic spatial reconstructor. New York Praeger .

[5] Higgins, W.E. Spyra, W.J.T. Karwosky, R.A. Ritman, E.L. (1991) A system for analyzing three-dimensional coronary angiograms. SPIE Med. Imaging V: Image Proc., 1445: 276-286.

[6] Saint-Felix, D. Trousset, Y. Picard, C. Rougee, A. (1990) 3D reconstruction of high contrast objects using a multi-scale detection / estimation scheme. In: K.H. Höhne, H. Fuchs, S.M. Pizer (eds.) 3D Imaging in Medicine, NATO ASI Series, F60: 147-158.

[7] Gerig, G. Kikinis, R. Jolesz, F.A. (1990 ) Image processing of routine spinecho MR Images to enhance vascular structures: comparison with MR Angiography. In: K.H. Höhne, H. Fuchs, S.M. Pizer (eds.) 3D Imaging in Medicine, NATO ASI Series, F60: 121-132.

[8] Vandermeulen, D. Delaere, D. Suetens, P. (1991) Image enhancement and soft segmentation of MR Angiography for an improved visualization of blood 
vessels. European Congress of Radiology (ECR'91), Vienna, abstract, supplement to European Radiology, 1: S 195.

[9] Trousset, Y. Schieber, D. Knoplioch, J. (1992) An algorithm for tracking vessels in three-dimensional angiograms. Proc. of the 14th IEEE-EMBS Conf, Paris: 2053-2054.

[10] Dillenseger, J.L. Hamitouche, C. Coatrieux, J.L. (1991) Visualisation d'images tridimensionnelles par lancer de rayons avec interpolation locale. Innov. et Tech. en Biol. et Med., 12, 3: 244-255.

[11] Sun, Y. (1989) Automated identification of vessel contours in coronary arteriograms by an adaptive tracking algorithm. IEEE Trans. on Med. Im., 8: $78-88$.

[12] Prokop, R.J. Reeves, A.P. (1992) A survey of moment-based techniques for unoccluded object representation and recognition. Computer Vision Graphics and Image Processing, 54: 438-460.

[13] Hu, M.K. (1962) Visual pattern recognition by moments invariant. IRE Trans. Information Theory, 8: 179-187.

[14] Luo, L. M. Hamitouche, C. Dillenseger, J.L. Coatrieux, J.L. (1993). A Moment Based Three Dimensional Edge Operator. IEEE trans. on BME, to appear.

[15] Reuzé, P. Dillenseger, J.L. Coatrieux, J.L. (1992) Detection et quantification de vaisseau en IRM par utilisation des moments géométriques 3D. Internal report. 


\section{CAPTIONS}

Figure 1 : The vessel model :

(a) initial coordinate system (E1) ;

(b) coordinate system after alignment on the $\mathrm{z}$ axis (E2).

Figure $2:$ Flow chart of the tracking algorithm.

Figure $3:$ Results on simulated data with $\mathrm{SNR}=5 \mathrm{~dB}$ and different windows sizes :

(a) rms error on the estimation of the orientation;

(b) mean error on the estimation of the radius.

Figure 4 : Results according to SNR for a given window size (73) :

(a) rms error on the estimation of the orientation;

(b) rms error on the estimation of the radius.

Figure 5 : Validation on real data from two view points :

MIP rendering (on the left);

3-D display of the extracted vessels (on the right).

Figure 6 : Comparison between the adaptive (left) and non adaptive (right) tracking scheme. 


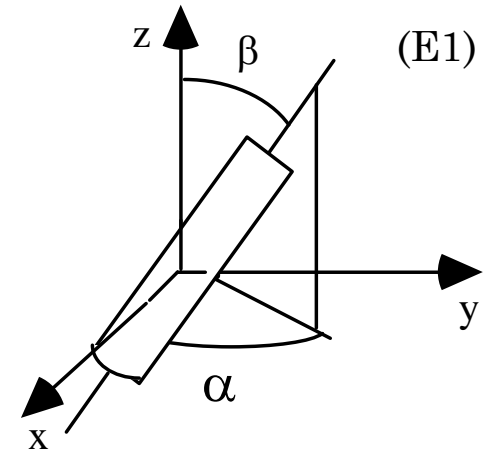

(a)

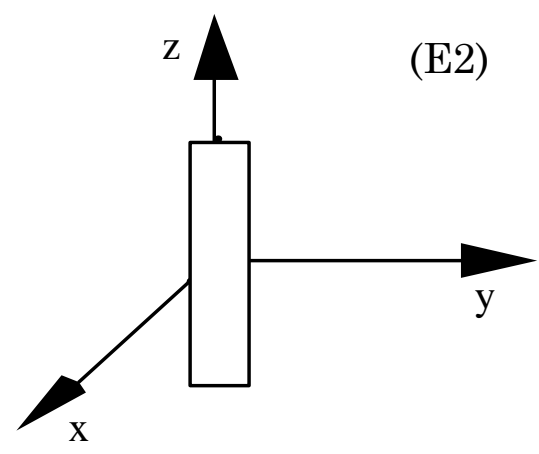

(b)

Figure 1 


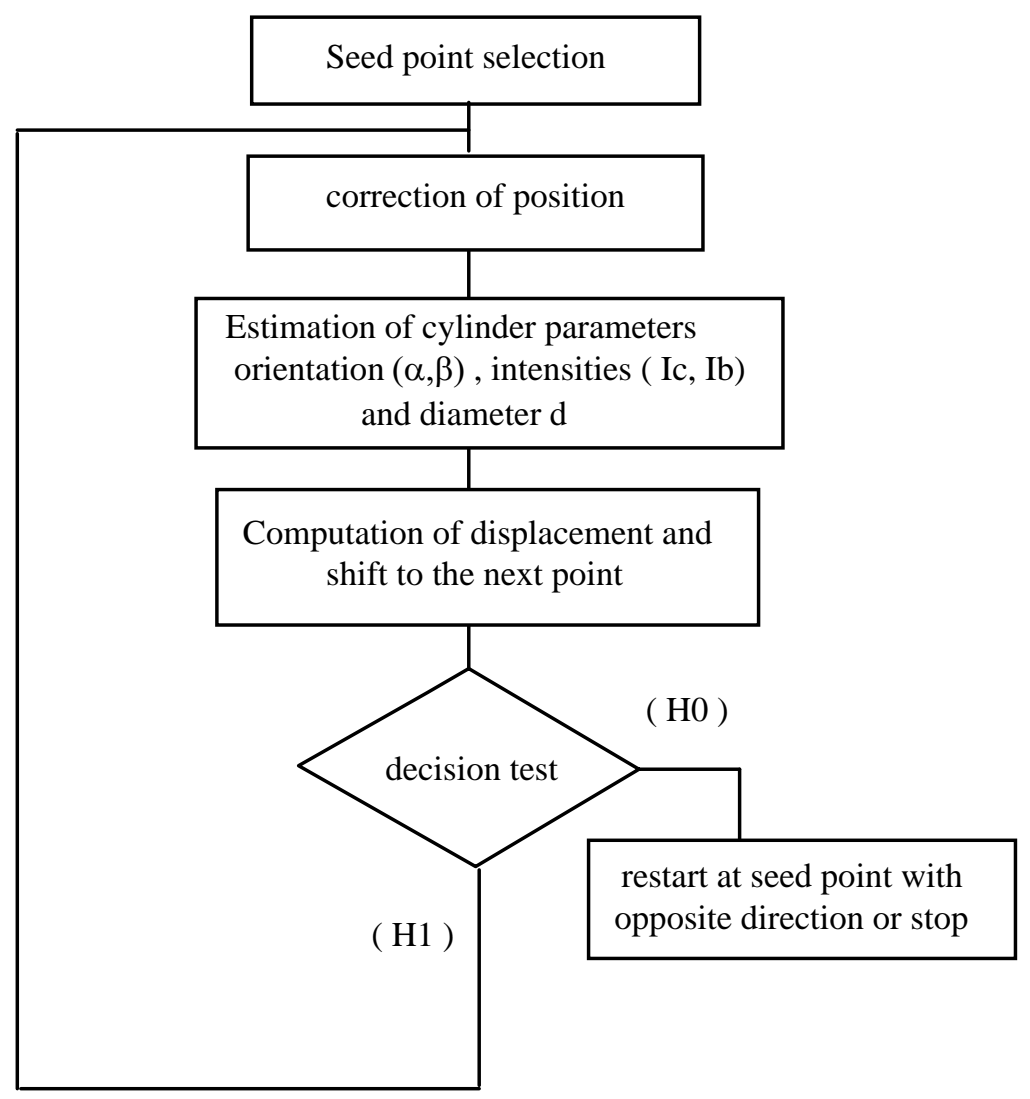

Figure 2 


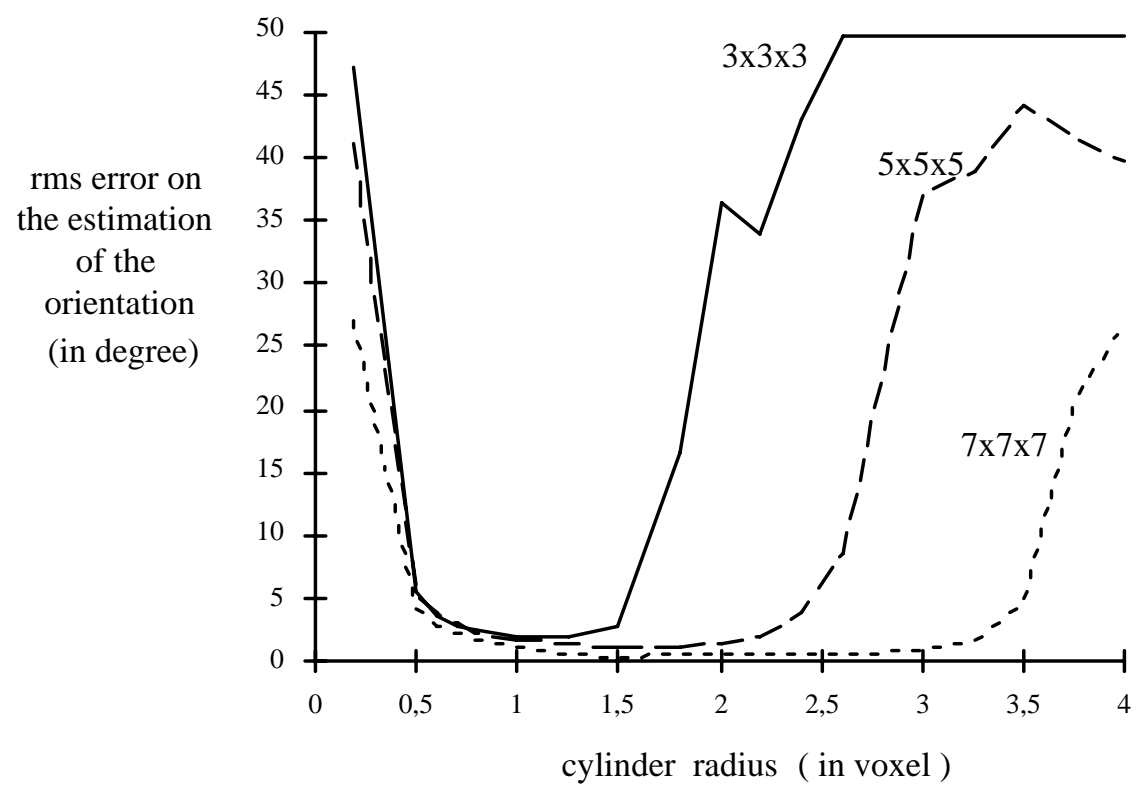

(a)

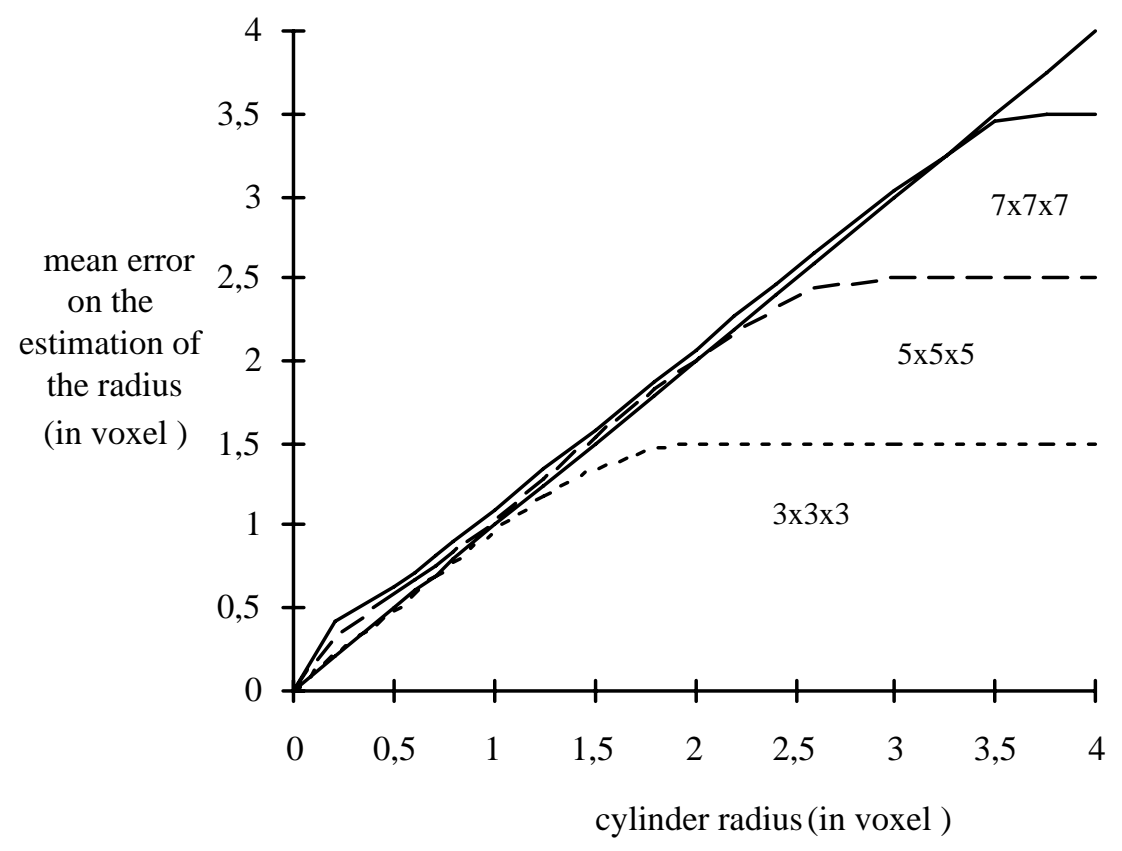

(b)

Figure 3 


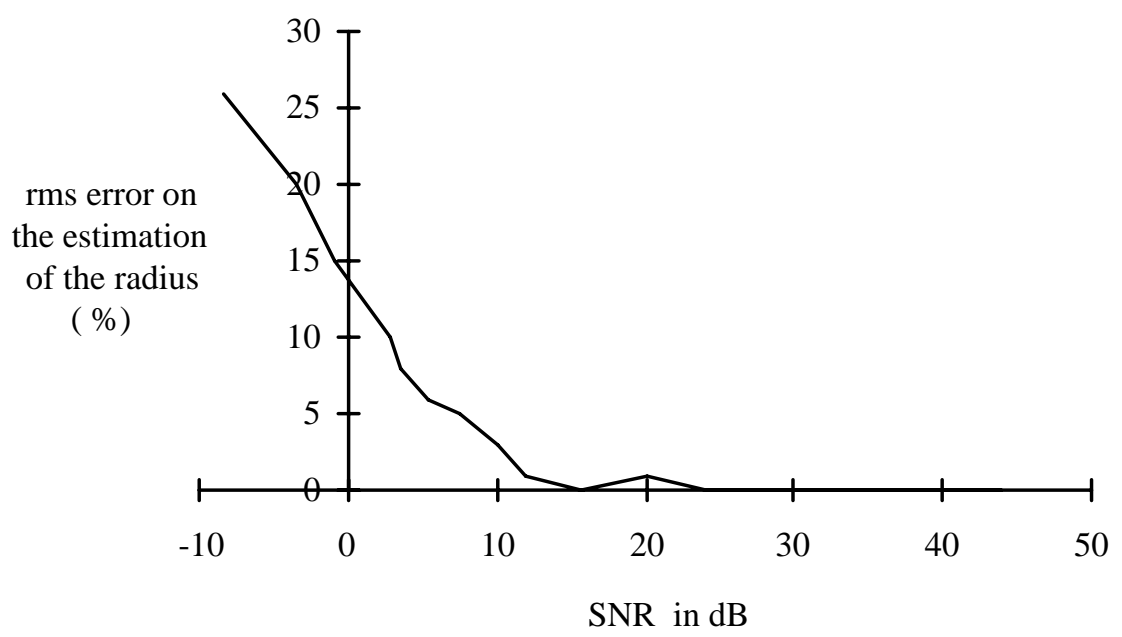

(a)

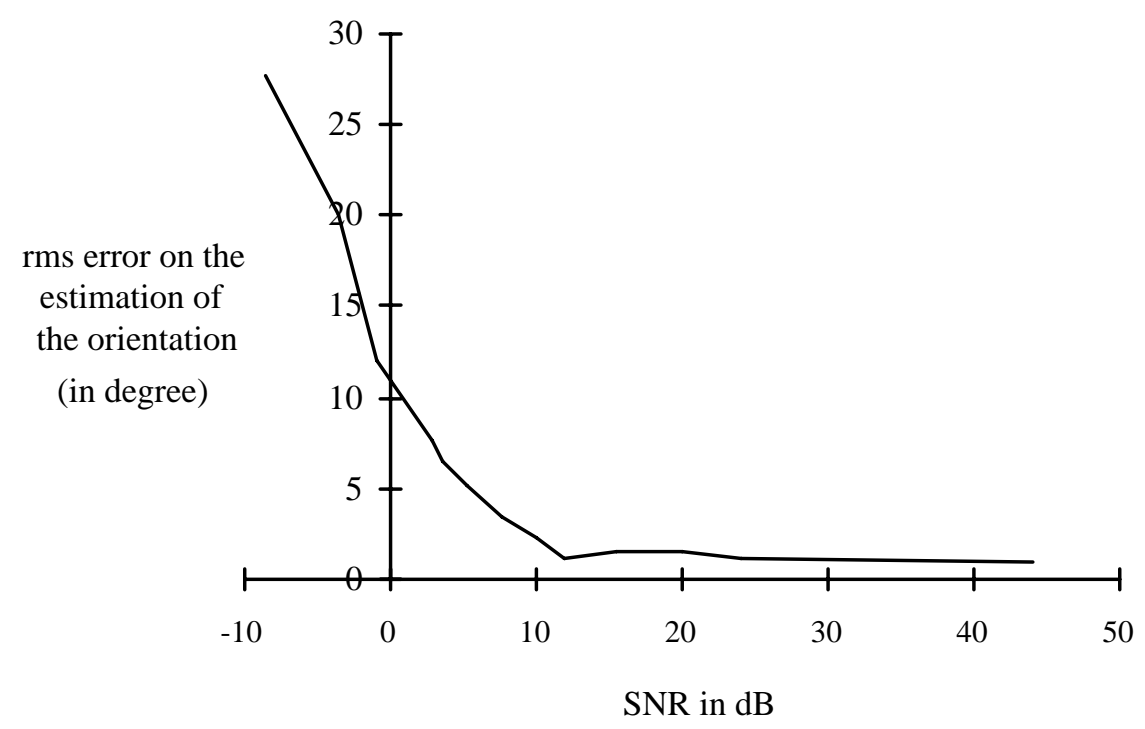

(b)

Figure 4 


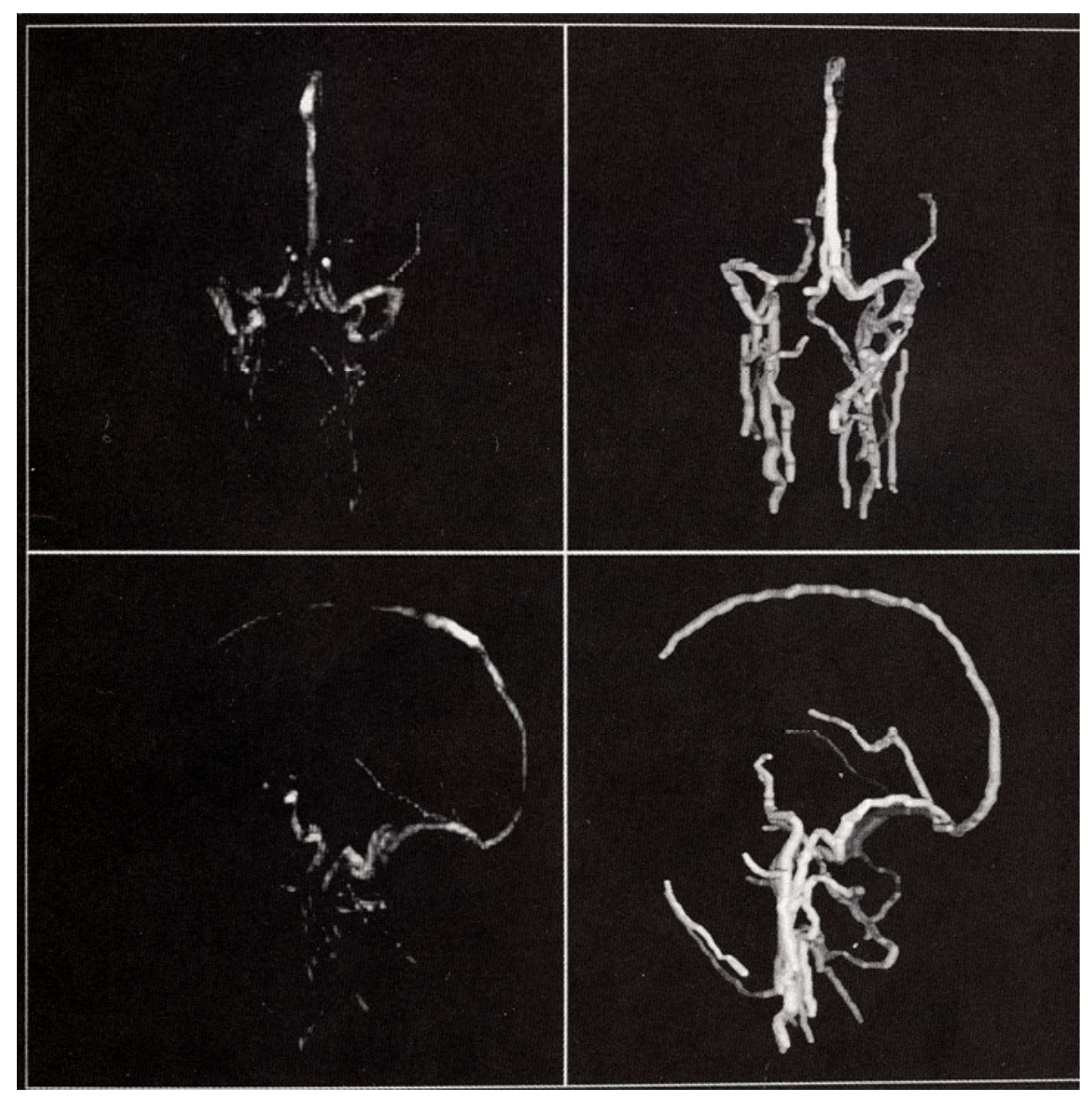

Figure 5 


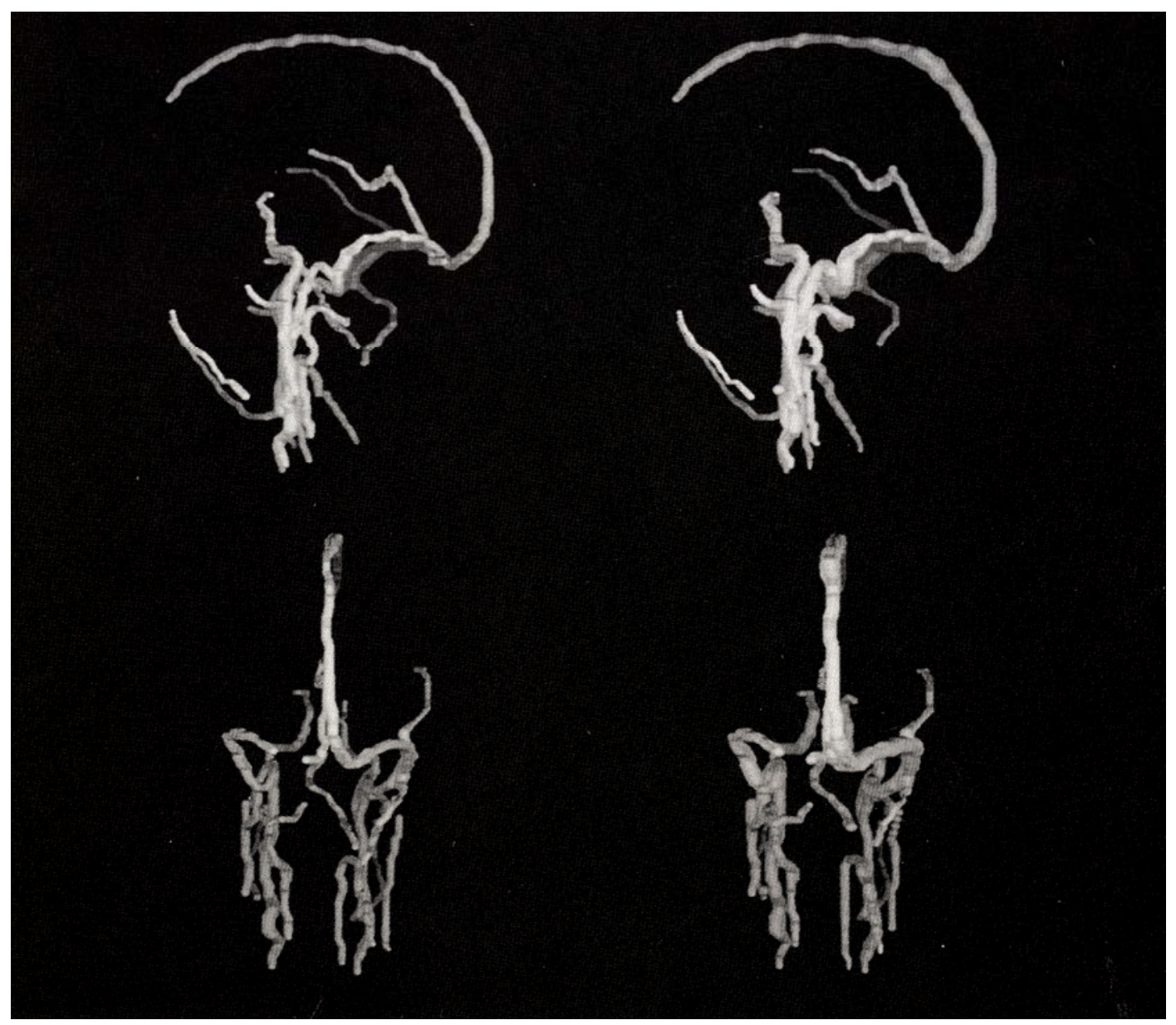

Figure 6 